\title{
Approche diachronique de la flore des bassins fermés halomorphes de la région d'Oran (N-O Algérie).
}

\author{
Diachronic analysis of the flora of the halomorphic closed basins in the region of Oran \\ (N-W Algeria)
}

Article reçu le 28 juin 2020 accepté le 5 octobre 2020

Kheïra BAHI ${ }^{1}$, Mohamed Djamel MIARA ${ }^{2 *}$, Seghir HADJADJ-AOUL $^{3}$

${ }^{1}$ Département d'Agronomie, Faculté des Sciences, Université Abelhamid Ibn Badis, Mostaganem, Algérie.

${ }^{2}$ Département et Faculté des Sciences de la Nature et du Vivant, Université Ibn Khaldoun, Tiaret, 14000, Algérie.

${ }^{3}$ Département de Biologie, Faculté des Sciences de la Nature et du Vivant, Université Ahmed Ben Bella 1, Oran, 31000, Algérie.

\section{Résumé}

Cette étude est une approche diachronique d'étude de la flore et de la végétation des zones humides de la région d'Oran.

Son but est de proposer une liste actualisée de la flore présente au niveau des ceintures des cinq bassins fermés d'Oran (la grande sebkha d'Oran, dayat Morsli, Oum El-Ghelaz, le lac Télamine et les salines d'Arzew). Cela nous permettra de d'identifier les changements que cette flore a subi et d'apprécier par conséquent la dynamique de la végétation locale à la lumière des conditions écologiques et anthropiques.

Nous avons réalisé 60 relevés phytoécologiques suivant un échantillonnage stratifié. Les espèces récoltées ont été identifiées et leurs noms actualisés ce qui a permis de dresser une liste actualisée de la flore régionale. Cette liste a été comparée à une autre liste plus ancienne datant de 50 ans.

La liste des taxons comporte 175 espèces relevant de 127 genres et de 39 familles. Le type biologique est dominé par des thérophytes de chorologie méditerranéenne. L'analyse diachronique des taxons retrouvés rapporte la présence de 80 espèces figurant dans les deux listes et la présence de 94 espèces $(55 \%)$ nouvelles.

Le nombre d'espèces tolérantes au sel reste relativement limité. Les ceintures de végétations étudiées connaissent une anthropisation importante due principalement au pâturage. La composition floristique a pu être également affectée par le changement climatique

Mots clés : végétation, zone humide, Halophytes, inventaire, évaluation, biodiversité, Oran.

\section{Abstract}

This study is a diachronic approach of the flora and vegetation of wetlands in the Oran region.

\footnotetext{
*Adresse électronique pour la correspondance : miara14130@yahoo.fr
} 
This study aim to provide an updated list of the flora present at the level of the belts of the five closed basins of Oran (the great sebkha of Oran, dayat Morsli, Oum El-Ghelaz, Telamine Lake and the saltworks of Arzew). This will allow us to identify the changes that this flora has undergone and consequently to appreciate the dynamics of the local vegetation in the light of ecological and anthropogenic conditions.

We carried out 60 phytoecological surveys using stratified sampling. The species collected were identified and their names updated, which made it possible to have an updated list of the regional flora. This list has been compared to another older list of 50 years old.

The list of taxa includes 175 species belonging to 127 genera and 39 families. The biological type is dominated by therophytes of Mediterranean chorology. Diachronic analysis reports the presence of 80 species appearing in the two lists as well as 94 new species (55\%).

The number of salt tolerant species remains relatively limited. The belts vegetation studied undergo significant anthropization mainly due to grazing. The plant composition could also be affected by climate change.

Keywords: vegetation, wetland, Halophytes, inventory, evaluation, biodiversity, Oran.

\section{Introduction}

Les zones humides méditerranéennes sont classées parmi les écosystèmes les plus riches de la planète (Médail \& Quézel, 1999) et abritent ainsi une biodiversité floristique remarquable. La végétation naturelle des zones humides en générale et la flore halophile en particulier sont bien connues au Maghreb. En effet, au Maroc, la flore des zones humides est estimée à plus de 670 espèces et sous-espèces regroupées en 83 familles (Hammada et al., 2004). Seule, la flore halophile compte environ 114 espèces et sous-espèces réparties entre 20 familles (Hammada et al., 2004). En Tunisie, la flore des zones humides est évaluée à 407 espèces regroupées en 228 genres et 75 familles (Ghrabi-Gammar et al., 2009).

En Algérie, 42 sites ont été classés Ramsar. Ils représentent une superficie de près de 3 millions d'hectares. Ces sites couvrent près de $50 \%$ de la superficie totale estimée des zones humides en Algérie (Anonyme, 2004). Parmi les auteurs qui se sont intéressés à la flore de ces zones humides nous citerons Géhu et al. (1994) concernant l'étude phytosociologique des groupements des lacs de la région d'El Kala, De Bélair (2005) qui a proposé la «Dynamique de la végétation des mares temporaires de la Numidie orientale (nord-est Algérie) », Ghezlaoui et al. (2011) qui ont effectué une analyse des peuplements halophytes dans le chott El Gharbi (Saîda). Egalement, Koull et al. (2013) dans la région de Biskra ont présenté la flore des zones humides de la vallée de l'oued Righ (Touggourt).

Parmi les auteurs qui se sont intéressés à la flore halophile de l'ouest algérien, Simonneau (1952) a présenté un catalogue de la flore halophile de la plaine de Mohamadia (anciennement appelée Perrégaux) comportant 486 espèces groupées en 286 genres et 77 familles. Par la suite, Quézel \& Simonneau (1960) ont examiné quelques aspects de la végétation des terrains salés des plaines sub-littorales de l'Oranie orientale. Enfin, les travaux de Dubuis \& Simonneau (1960) ayant 
porté sur les halophytes des bassins fermés du plateau d'Oran sont également à souligner. Ces deux auteurs ont proposé une liste comportant 180 espèces relevant de 136 genres et 36 familles. Dans ce travail, nous nous proposons de dresser une liste actualisée de la flore halophile des bassins fermés de la région d'Oran. Pour évaluer la dynamique de cette végétation halophyte, notre liste sera comparée à celle de Dubuis \& Simonneau (1960). Aussi, nous tenterons de réaliser une interprétation de nos résultats en relation avec les conditions écologiques locales (climat, salinité et anthropisation) qui sont susceptible d'être à l'origine d'une éventuelle variation de la composition floristique de ces milieux.

\section{Matériels et méthodes}

\subsection{La Région d'étude}

La région d'Oran est située au nord-ouest de $1^{\prime}$ Algérie. Elle est comprise entre $1^{\circ} 03^{\prime}$ et $0^{\circ} 15^{\prime} \mathrm{W}$ de longitude ouest et entre $35^{\circ} 27$ et $35^{\circ} 45$ de latitude nord (Figure 1). C'est une dépression qui suit l'orientation générale sud-ouest nord-est des reliefs et qui comporte cinq bassins fermés (la grande sebkha d'Oran, daya Morsli, Oum El-Ghelaz, le lac Télamine et les salines d'Arzew). Le lac Télamine et les salines d'Arzew sont situés dans une dépression appelée plateau d'Oran. Les bassins fermés d'Oran peuvent être considérés comme des témoins des formations lagunaires et lacustres caractéristiques de la topographie et l'hydrographie du Pliocène et du début de l'ère quaternaire (Thomas, 1976).

La carte des sols de Durant (1954) indique que les zones prospectées sont couvertes par un sol de type Solontchak bordé par des sols alluviaux.

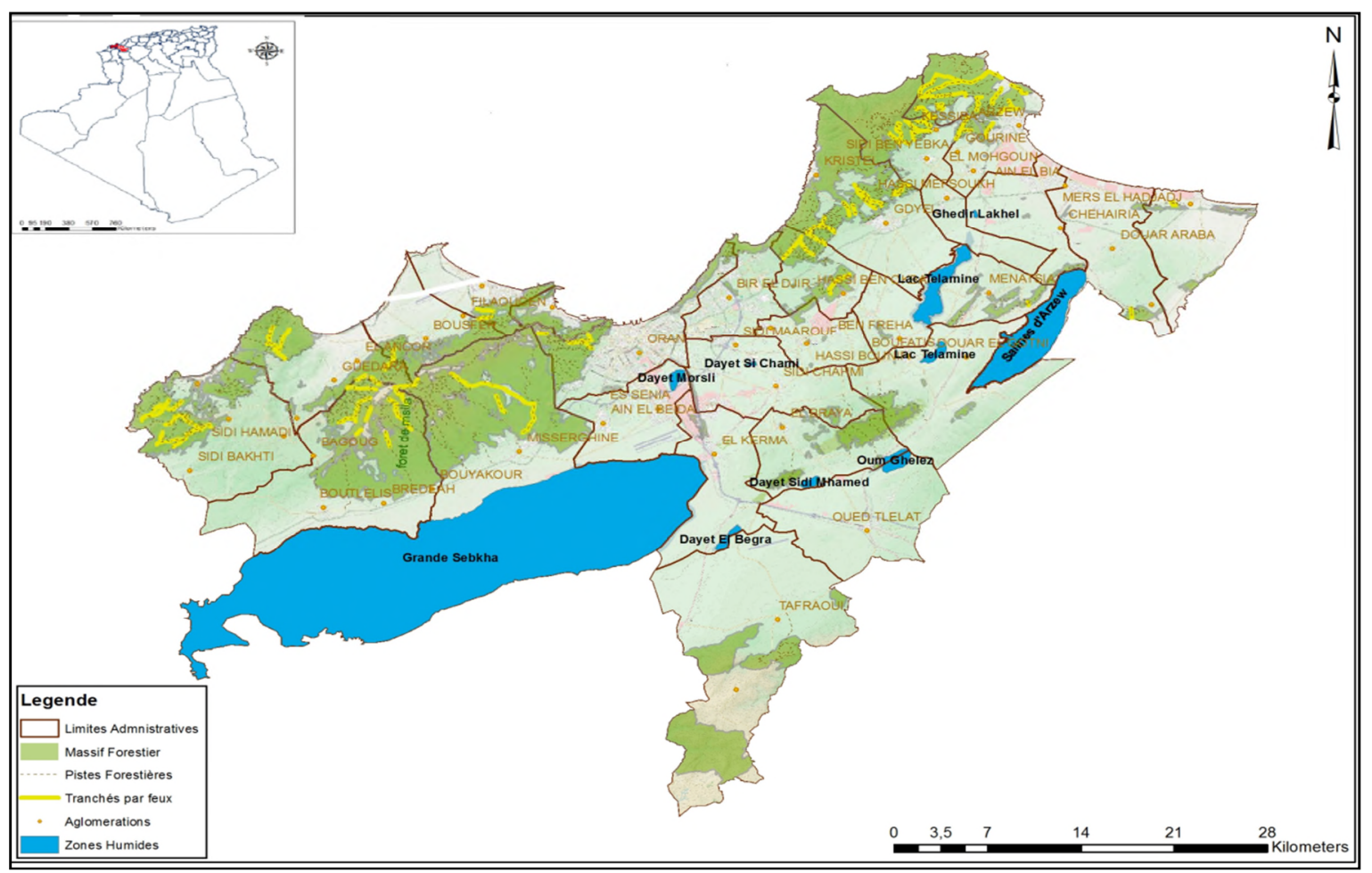

Figure 1 : Localisation des zones humides de la région d'Oran. 


\subsection{Analyse climatique}

Le climat général de la région est de type méditerranéen, caractérisé par des étés secs et chauds et des hivers doux et pluvieux recevant la presque totalité des pluies. Pour mettre en évidence l'importance du changement climatique de notre région, nous présenteront une analyse des données climatiques (précipitation et température) de Seltzer (1946) pour la période de 1913-1936 qui sera comparée à celle de (1987-2012). Ces dernières données sont fournies par l'Office national de la météorologie.

\subsection{Relevés phytoécologiques}

$\mathrm{Au}$ total, 60 relevés phytoécologiques ont été envisagés à travers cette région selon un échantillonnage stratifié. Nous avons effectué nos relevés pendant les années 2012 à 2015 durant la période printanière où la phénophase est optimale pour les déterminations botaniques. Nous avons procédé à l'identification des spécimens à l'aide des flores de Quézel et Santa (1962-63), Ozenda (1977), Maire (1957), avec la flore de l'Andalousie (Blanca et al., 2009) ainsi qu'avec la flore pratique du Maroc (Fennane et al., 1999). En plus de ces flores, nous avons utilisé le travail d'Aimé \& Roiron (1985) pour le genre Limonium de la région d'Oran ainsi que l'herbier du laboratoire de Botanique de l'université d'Oran. L'actualisation de la nomenclature ancienne a été réalisée à l'aide de l'index synonymique de Dobignard \& Chatelain (2010-2013), ainsi des plateformes en ligne «Base de données des plantes d'Afrique» (Conservatoire et Jardin botanique de la ville de Genève, 2012) et « World Flora Online » (2020).

\section{Résultats et discussion}

\subsection{Richesse floristique}

L'identification des taxons observés a permis de reconnaitre 175 espèces appartenant à 127 genres et 39 familles. Parmi les familles citées, quatre sont dominantes notamment les Asteraceae (31espèces, 19\%), les Poaceae (28 espèces, 17\%), les Amaranthaceae (14 espèces, 9\%) et les Fabaceae (10 espèces, 6\%) (Figure 2).

La composante floristique de nos sites d'étude représente de $4 \%$ de la flore totale de l'Algérie septentrionale (175/4125). Ce taux est considéré comme négligeable au vue de la superficie des sites parcourus, mais non négligeable lorsqu'on pense que ces milieux halomorphes sont le plus souvent considérés comme stériles.

\subsection{Traits fonctionnels des taxons}

\subsubsection{Les types biologiques}

La répartition des types biologiques des espèces rencontrées est caractérisée par le schéma suivant Thérophytes $>$ Hémichryptophytes $>$ Géophytes $>$ Chaméphytes $>$ Nanophanérophytes $>$ 
Phanérophytes (Figure 3). Globalement, les mêmes proportions ont été observées par Aidoud (1983) dans les steppes halophiles de l'intérieur.

La dominance des thérophytes avec une centaine d'espèces ( $60 \%$ de l'effectif total) dénote d'une thérophytisation avérée ce qui constitue une réelle menace pour la flore originelle de ces milieux (Miara et al., 2016).

D'après Hammada et al (2004), cette dominance de thérophytes pourra être interprétée par le fait de la présence des habitats à immersion saisonnière qui sont plus favorables au développement de plantes annuelles à germination et croissance rapides. La plupart de ces thérophytes éphémères disparaissent aves les premières chaleurs de l'été. Nous avons remarqué également qu'en plein été la végétation de ces régions change complètement de couleur et vire au rouge et au marron (couleur observée en particulier dans les genres Arthrocnemum, Salicornia et Suaeda), et que seules les vrais halophytes résistent aux remontées des sels comme les Amaranthaceae ou les Plumbaginaceae. Aux premières pluies la couleur verte revient annonçant l'installation des thérophytes.

\subsubsection{Chorologie des taxons}

Nous avons compté 108 espèces appartenant à l'ensemble méditerranéen dont 65 espèces sont typiquement méditerranéennes ainsi que 38 espèces appartenant à l'ensemble septentrional et 32 de large répartition (Figure 4). Par ailleurs, notre liste compte 5 espèces endémiques dont trois espèces de Limonium. Il s'agit de Limonium cossonianum Kuntze endémique de la sebkha et des bords de mer de l'Oranie (O1-2), L. cymuliferum (Boiss.) Sauv. \& Vindt endémique de la sebkha et des terrains salés d'Oranie (O2-3), L. duriaei (de Girard) Kuntze endémique des sebkhas (lac salés occupant le fond d'une dépression) et des haut- plateaux algérois et oranais ( $\mathrm{O} 2, \mathrm{H} 1)$, Spergularia doumerguaei P. Monnier endémique du nord-ouest de l'Afrique, présente uniquement aux environs de la Sebkha d'Oran et à la Macta (O1), et Sonchus mauritanicus Boiss. \& Reut. endémique d'Afrique du nord (Quézel \& Santa, 1962-63). Ces espèces considérées comme endémiques de l'Algérie par ces derniers auteurs, ont vraisemblablement changé de statut chorologique. Les 3 espèces du genre Limonium: Limonium cossonianum Kuntze, L. cymuliferum (Boiss.) Sauv. \& Vindt, L. duriaei (de Girard) Kuntze, ainsi que Spergularia doumerguaei P. Monnier sont actuellement considérées comme endémiques algéromarocaines alors que Sonchus mauritanicus Boiss. \& Reut. est désormais reconnu comme une endémique du Maghreb (Maroc, Algérie, Tunisie) d'après El Oualidi et al. (2012).

En plus de l'endémisme, certaines plantes présentent également un intérêt patrimonial. Ces taxons sont protégés par la législation algérienne portée par le Décret exécutif $\mathrm{n}^{\circ}$ 12-03 (2012) fixant la liste des espèces végétales non cultivées protégées. Tel que Limonium cossonianum Kuntze ou (L. gummiferum Dur. Kuntze), L. virgatum Willd. Kuntze, Salicornia patula DuvalJouve et Spergularia doumerguaie P. Monnier (Journal officiel ,2012).

Parmi les classes de fréquences des espèces proposées par Quézel et Santa (1962-63), nous avons noté certaines espèces assez communes ayant une large répartition mondiale. En Algérie leur faible présence est limitée à l'Oranie (O1-2). C'est le cas de 18 espèces présentes dans notre inventaire, notamment: Anisantha fasciculata (C. Presl) Nevski, Arenaria emarginata Brot., Beta macrocarpa Guss., Chenopodium glaucum L. Erodium ciconium (L.) L'Hér. Erodium laciniatum (Cav.) Willd, Limonium cossonianum Kuntze, L. cymuliferum (Boiss.) Sauv. \& 
Vindt, L. sinuatum subsp. sinuatum Sauvage \& Vindt., Lycium intricatum Boiss., Malva hispanica L., Mesembryanthemum cristalinum L., Phalaris paradoxa L., Salicornia patula Duval-Jouve, Sarcocornia perennis (Mill.) A.J. Scott, Senecio leucanthemifolius subsp. mauritanicus (Pomel) Gre., Silene nicaeensis All, et Suaeda maritima (L.) Dumor.

En ce qui concerne la rareté et la vulnérabilité de certaines espèces, nous avons reconnu 11 taxons considérées comme rares en Oranie: Anisantha fasciculata (C. Presl) Nevski, Chenopodium glaucum L, Malva neglecta Wallr., Malva rotundifolia L, Limonium virgatum (Willd.) Fourr., L. duriaei (de Girard) Kuntze, L. sinuatum subsp. sinuatum Sauvage \& Vindt., Salicornia patula Duval-Jouve, Scorpiurus muricatus (L.) subsp. muricatus Thell., Spergularia doumerguaei P. Monnier, Sonchus mauritanicus Boiss. \& Reut.

À celles-ci, s'ajoutent 21 espèces communes dans nos régions qui sont considérées comme rares ailleurs : Anisantha madritensis (L.) Nevski, Atriplex patula L., Atriplex prostrata DC., Beta macrocarpa Guss., Dittrichia viscosa (L.) Greuter, Oxalis pres-caprae L., Medicago polymorpha L., Rumex pulcher L., Sinapis arvensis L., Rostraria cristata (L.) Tzvelev, Salsola kali L., Sonchus mauritanicus Boiss. \& Reut, Elytrigia repens (L.) Desv. ex Nevski, Limonium echioides (L.) Mill., Cynomorium coccineum L. subsp. coccineum, Trifolium tomentosum L., Silene nicaeensis All., Suaeda maritima (L.) Dumort., Sinapis arvensis L., Melilotus indicus (L.) All., Silene gallica L. (Quézel \& Santa, 1962-63).

\subsection{Approche diachronique}

La liste floristique des bassins fermés que nous présentons regroupe la grande Sebkha d'Oran, daya Morsli ainsi que les bassins du plateau d'Oran qui ont été étudiés auparavant par Dubuis \& Simonneau (1960). La surface de notre région d'étude est beaucoup plus étendue que celle parcourue par ces derniers auteurs. Toutefois le catalogue qu'ils ont présenté comportait 180 espèces regroupées en 136 genres et 36 familles. Pour les mêmes sites, nous n'avons observé que 135 espèces, 114 genres appartenant à 39 familles.

Afin de pouvoir effectuer cette comparaison, nous avons tenté d'actualiser la liste ancienne par le biais de l'index synonymique de Dobignard \& Chatelain (2010-13). La comparaison diachronique des données floristiques rapporte que 80 espèces (45\%) figurent dans les deux listes, alors que 94 espèces (55\%) n'y figurent pas (Tableau 2 - voir fin de l'article)

Certaines espèces caractéristiques de la région non pas été retrouvées lors de la réalisation des relevés en raison de la méthode d'échantillonnage non systématique. C'est le cas d'Halopeplis amplexicaulis (Vahl.) Ung.-Stern (halophyte, annuelle) observée par Dubuis \& Simonneau en 1960, puis signalée par Tremblin (2000) aux salines d'Arzew ainsi que par Aboura (2011) dans les environs de Misserghin. Ce taxon a été retrouvé en quelques pieds en juillet 2018 au niveau des salines d'Arzew lors d'une recherche subjective. Par ailleurs, certaines espèces n'ont pas été retrouvées à l'image d'Ammochloa pungens (Schreb.) Boiss. et Stipa retorta Cav, ce qui pourrai être en relation avec le surpâturage très fréquent dans ces régions.

Néanmoins, certains taxons figurant dans notre catalogue ne sont pas connus pour ces régions, à l'image de Mesembryanthemum cristallinum L., Atriplex patula L., Salsola kali L. et Chenopodium album L. Ces taxons sont en effet absents dans la liste de Dubuis \& Simonneau (1960). Ces auteurs ont également cité des espèces comme Chamaerops humilis L. et Pistacia lentiscus L. qui sont présents dans notre région mais assez loin des ceintures halomorphes. 
D'autres espèces ont été retrouvées accidentellement comme Atriplex canescens (Pursh) Nutt. avec un seul pied observé au niveau du lac de Telamine. Par ailleurs, nous avons noté la dominance des Amaranthaceae dans la liste des taxons. En effet, des genres comme Arthrocnemum, Atriplex, Salicornia et Suaeda connaissent une très large extension au niveau de ces écosystèmes halomorphes bien que leur nombre d'espèces soit très limité par rapport aux Poaceae et aux Asteraceae.

Cette végétation frutescente reste très spécifique de ces milieux halomorphes. En effet, la tolérance au sel est le facteur décisif qui détermine l'installation, le maintien, l'extension ou la disparition de ces espèces halophiles (Simonneau, 1952). Malgré cette adaptation, le nombre d'espèces tolérant le sel reste relativement limité.

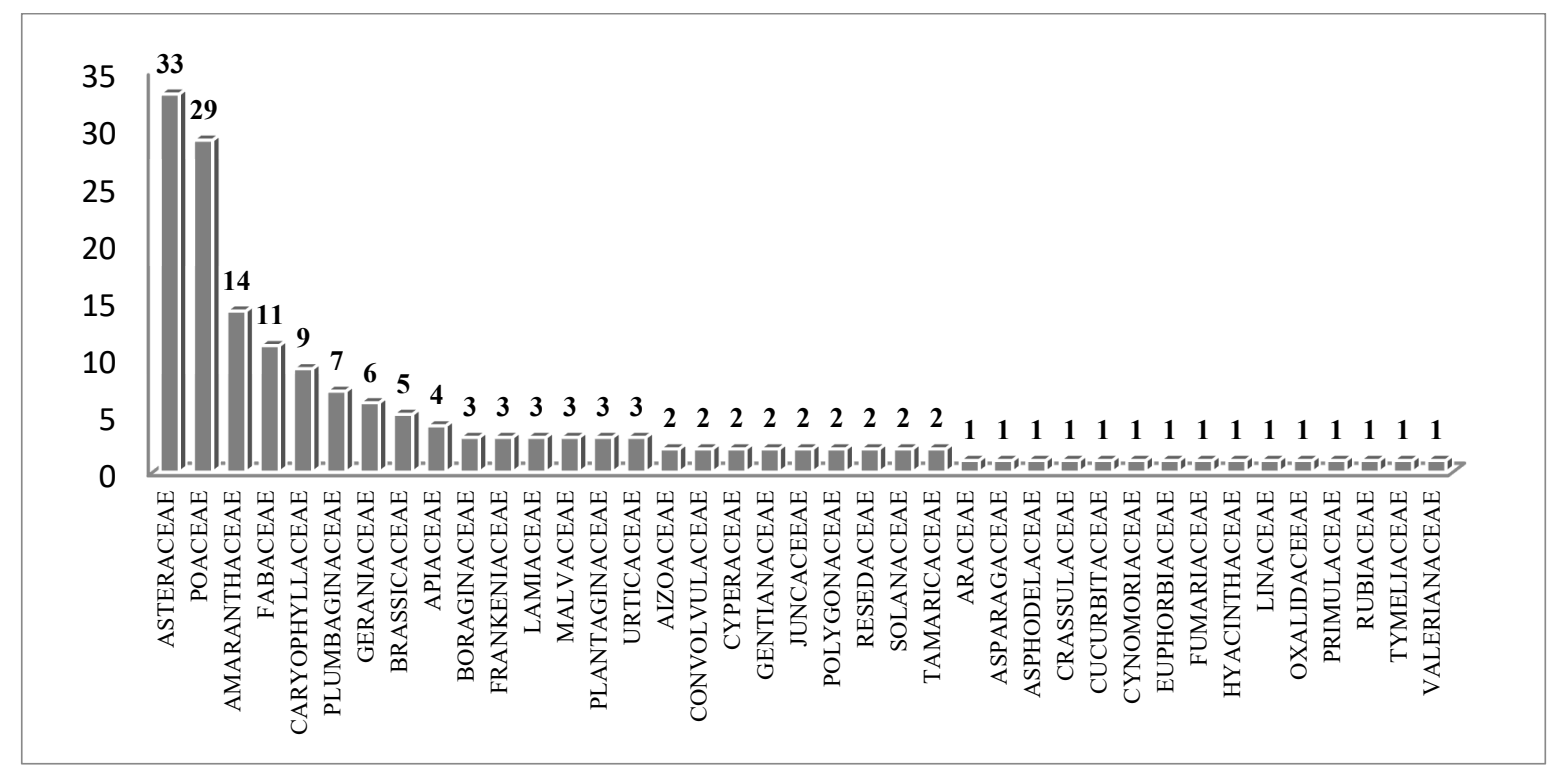

Figure 2 : Les familles dominantes (nombre des espèces).

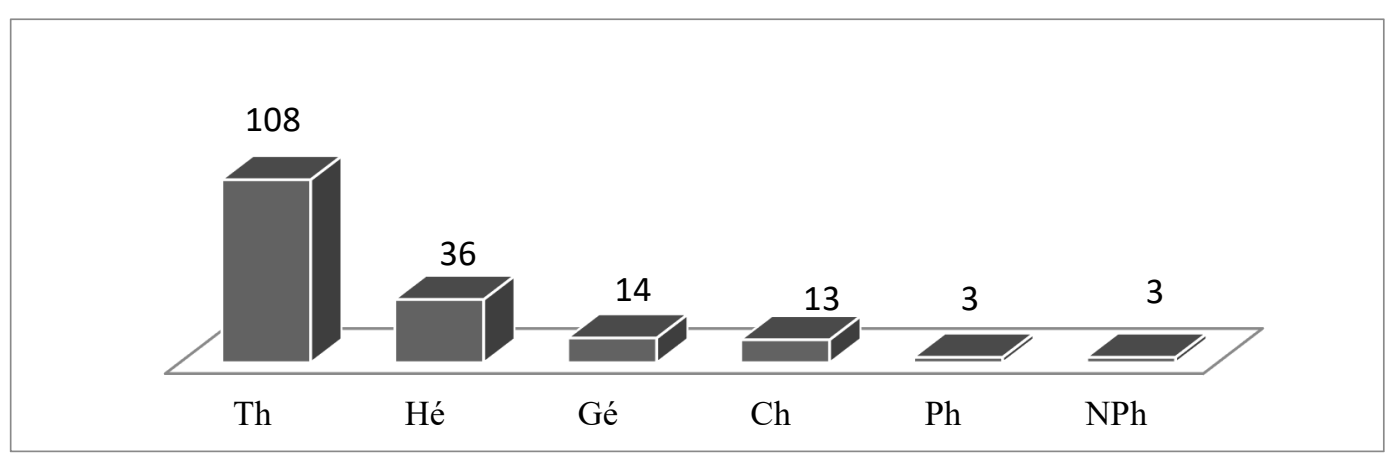

Figure 3 : Le spectre Biologique (nombre des espèces).

Thérophytes (Th), Hémicryptophytes (He), Géophytes (Gé), Chaméphyte (Ch), Nanophanérophytes (NPh) Phanérophytes (Ph). 


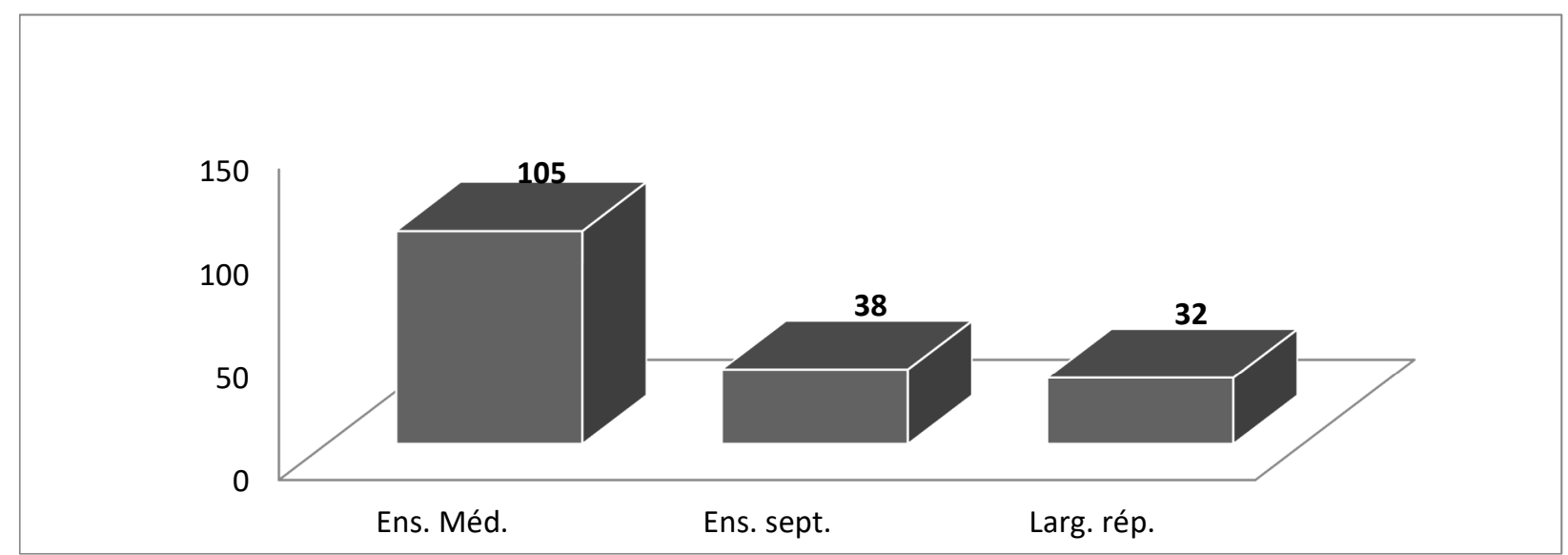

Figure 4 : Le spectre Biogéographique (nombre des espèces).

Ens. Méd. : Ensemble méditerranéen

Ens. Sept. : Ensemble septentrionale

Larg. rép. : Large répartition).

\section{Analyse climatique}

La comparaison des données climatiques entre les deux périodes étudiées (1913-1938 et 19872012) montre une diminution des précipitations accompagnée d'une augmentation des moyennes de température pour la période plus récente par rapport à celle plus ancienne. Ainsi, la saison sèche s'étend sur cinq à six mois au lieu d'environ 4 mois auparavant. Cette analyse diachronique (Figure 5; Tableau 1) montre que la région d'Oran est passée du niveau bioclimatique subhumide à hiver chaud au niveau bioclimatique semi-aride à hiver tempéré. Les mêmes résultats ont été obtenus par Miara et al. (2013) dans la région de Tiaret.

Tableau 1 : Analyse du climat (ancien-récent) d'Oran.

\begin{tabular}{|c|c|c|c|c|c|c|}
\hline Périodes & $\begin{array}{c}\text { Tmax } \\
\left({ }^{\circ} \mathbf{C}\right)\end{array}$ & $\begin{array}{c}\text { Tmin } \\
\left({ }^{\circ} \mathbf{C}\right)\end{array}$ & $\begin{array}{c}\text { CPann } \\
(\mathbf{m m})\end{array}$ & $\mathbf{Q 2}$ & $\begin{array}{c}\text { Niveau } \\
\text { bioclimatique }\end{array}$ & Hiver \\
\hline \hline $1913-1938$ & 28,7 & 9,1 & 520 & 90 & $\begin{array}{c}\text { subhumide } \\
\text { inférieur }\end{array}$ & chaud \\
\hline $1987-2012$ & 32,9 & 5,1 & 325,7 & 40,1 & $\begin{array}{c}\text { semi-aride } \\
\text { inférieur }\end{array}$ & tempéré \\
\hline
\end{tabular}

(Tmax : température maximale, Tmin : température minimale CPann : Cumul de précipitations annuelles, Q2 : Quotient d'Emberger) 


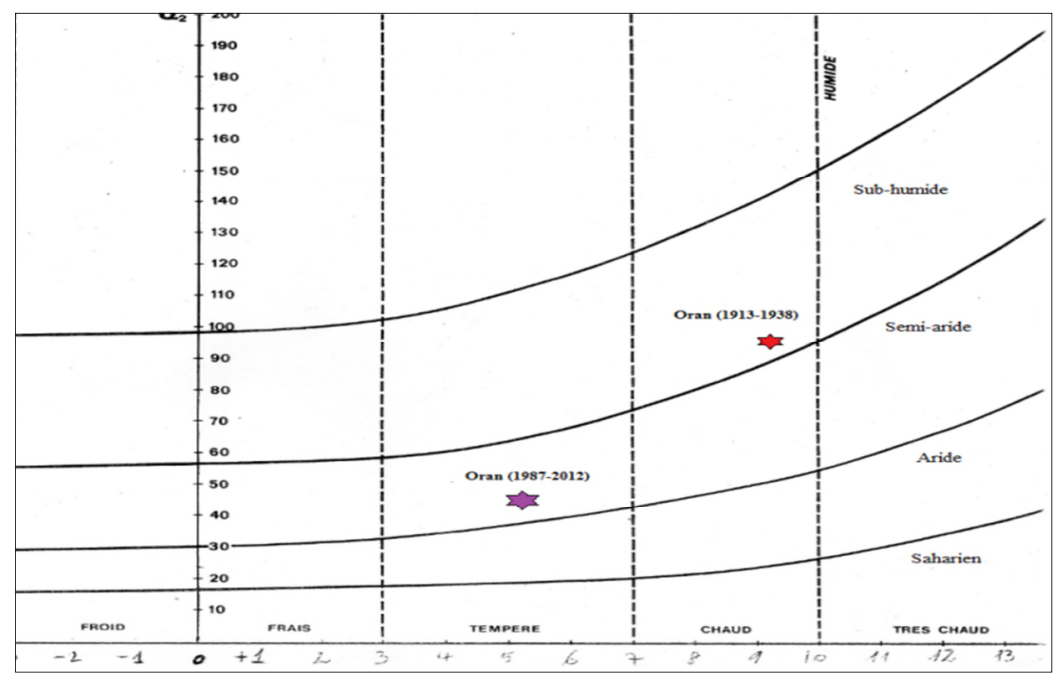

Figure 5 : Climagramme d'Emberger de la région d'Oran ( 5 1913-1938, 1987-2012)

\section{Les facteurs agissant sur la biodiversité}

Parmi les facteurs qui ont certainement contribué à la variation constatée de la composition floristique de la flore halophile des zones humides d'Oran, en plus de la salinité, nous retenons deux facteurs principaux qui sont le changement climatique et l'activité humaine.

\subsection{Le changement climatique}

Le climat est un paramètre important dans l'installation, du maintien ou de l'extinction des espèces. Comme le montrent les résultats obtenus suite à l'analyse climatique diachronique des données régionales, les baisses de précipitations, l'augmentation des moyennes de températures ainsi que la durée de saison sèche dans la région d'Oran, qui ont provoqué le passage du niveau sub-humide au niveau semi-aride de cette région, sont vraisemblablement à l'origine de cette modification floristique. En effet, une aridité climatique est susceptible de favoriser une thérophytisation massif des milieux naturels en région méditerranéenne (Bammi et al., 2004 ; Miara et al., 2013)

\subsection{L'anthropisation (pâturage)}

Les zones humides d'Oran ont depuis longtemps été l'endroit privilégié de pâture des ovins et des bovins de la région. Selon Loisel \& Gomilla (1993), il est possible de déterminer le degré de la perturbation induite par la pression anthropique sur les différents groupements par le calcul de l'indice de perturbation :

\section{IP = Nb Cham + Nb Th / le nombre total des espèces}


Avec $\mathrm{Nb}$ Cham. $=$ Nombre de chaméphyte, $\mathrm{Nb}$ Th. $=$ Nombre de thérophytes. Cet indice est de $65,47 \%$, ce qui indique une forte thérophytisation. Cette dernière implique une dégradation de la végétation qui est due à une anthropisation intense (Hachemi et al, 2012; Miara et al, 2016).

\section{Conclusion}

Cette étude a permis d'identifier 175 taxons pour la plupart halophiles (halophytes vraies et facultatives), appartenant à 127 genres et 39 familles. Parmi ceux-ci, nous avons noté 36 espèces rares ou assez rares et 5 endémiques. Les familles des Asteraceae et Poaceae sont les mieux représentées. Avec un lot important de thérophytes, le spectre biologique semble être typique de l'ambiance bioclimatique semi-aride, ce qui a été également observé sur le plan climatique régional. Par ailleurs, cette thérophytisation a vraisemblablement aussi une autre origine qui srait le pâturage, très intense dans ces régions.

Il s'avère ainsi que ces deux facteurs (changement climatique et surpâturage) ont joué un rôle important dans la variation floristique observée au niveau de ces bassins fermés. Les longues saisons sèches associées aux remontées de sel ainsi que le broutage continu dans l'espace et dans le temps ont affecté certainement leur extension et composition floristique. Les espèces citées par Dubuis \& Simonneau (1960) qui n'ont pas été retrouvées à l'issue de la présente étude devraient faire l'objet de sérieuses réflexions quant à leur existence et à leur statut de conservation.

Ces zones humides de la région d'Oran, qui jouent un rôle important dans le maintien d'une biodiversité floristique et faunistique importante, méritent une protection et une conservation digne de leurs classements dans la convention Ramsar.

\section{Références}

Aboura R., 2011. Contribution à l'étude des Atriplexaies en Algérie occidentale, aspects physionomiques et phytodynamique.- Thèse Doct. Univ. Tlemcen, $156 \mathrm{p}$.

Aimé S. \& Roiron P., 1985. Les espèces vivaces du genre Limonium en Oranie (Algérie). Candollea. 40, 409-423.

Aidoud A., 1983. Contribution à l'étude des écosystèmes steppiques du Sud-Oranais phytomasse, productivité primaire et applications pastorales. Thèse Doct. 3e cycle. Univ. Sci. Tech. H. Boumediene, Alger, 245 p. +ann.

Antipolis., 2003. Les menaces sur les sols dans les pays méditerranéens : Etude bibliographique ; Plan Bleu Centre d'activités régionales.

Anonyme., 2004. Atlas des zones humides algériennes d'importance internationale.- Ministère de l'Agriculture et du Développement Rural, Direction Générale des Forêts. 75 p.

Bammi J. \& Douira A., 2004. Contribution à la connaissance de la flore vasculaire de la forêt de l'Achach, plateau central (Maroc), Acta Botanica Malacitana. 29: 23-41. 
Blanca G., Caezudo B., Cueto M., Fernandez Lopez C. \& Torres M., 2009. Flora Vascular de Andalucía Oriental.4 volumes, Edita: Consejería de Medio Ambiente. Junta de Andalucía.

Conservatoire et Jardin botanique de la ville de Genève, 2012. Base de données des plantes d'Afrique, https://www.ville-ge.ch/musinfo/bd/cjb/africa/recherche.php?langue=fr, consulté le $13 / 10 / 2020$

Daget P., 1984. Introduction à une théorie générale de la méditerranéité. Bulletin de la Société Botanique de France. 131 (2/3/4), 31-36.

De Bélair G., 2005. Dynamique de la végétation de mares temporaires en Afrique du Nord (Numidie orientale, NE Algérie). Journal of Mediterranean Ecology. Vol. 31, No. 1, 83-100.

Dubuis A. \& Simonneau P., 1960. Contribution à l'étude de la végétation halophile des bassins fermés du plateau d'Oran. Direction de l'hydraulique et de l'équipement rural. ClairboisBirmondreis. Alger. 114 p.

Dobignard A. \& Chatelain C., 2011-2013. Index synonymique de la Flore d'Afrique du nord. Conservatoire et jardin botanique de Genève. 5 volumes.

Durant JH., 1954. Sols de l'Algérie.- Publications du Service de la Colonisation et de l’Hydraulique. Pédologie. №2, Alger. 244 p.

El Oualidi J., Khamar H., Fennane M., Ibn Tattou M., Chauvet S. \& Sghir Taleb M., 2012. Checklist des endémiques et spécimens types de la flore vasculaire de l'Afrique du Nord.Documents de 1'Institut Scientifique de Rabat. N²51, 89p.

Emberger L., 1971. Travaux de botanique et d'écologie. Ed. Masson et Cies. 520p.

Fennane M., Ibn Tattou M., Mathez J., Ouyahya A. \& El Oualidi J., 1999. Flore Pratique du Maroc. Manuel de détermination des plantes vasculaires. 3 Volumes. Travaux de l'Institut Scientifique, série Botanique, $n^{\circ} 36,1999$, Rabat.

Hachemi N., Hassnaoui O., Benmehdi I., Medjati N. \& Bouazza M., 2012. Contribution à l'étude de la thérophytisation des matorrals des versants sud des monts de Tlemcen (Algérie occidentale). Mediterranea. N²3, 158-180.

Hammada S., M. Dakki, M. IbnTattou, Ouyahya A. \& Fennane M., 2004. Analyse de la Biodiversité floristique des zones humides du Maroc. Flore rare menacée et halophile.

Géhu JM., Kaabeche M. \& Gharzouli R., 1994. Phytosociologie et typologie des rives des lacs de la région d'El-Kala (Algérie). Colloque de Phytosociologie : XXII, Bailleul.

Ghezlaoui BD., Benabadji N., Benmansour D. \& Merzouk A., 2011. Analyse des peuplements végétaux halophytes dans Le chott el-Gharbi (Oranie-Algérie) -Acta Botanica Malacitana 36.113-124. 
Ghrabi-Gammar Z., Daoud-Bouattour A., Ferchichi H., Gammar A.M., Muller S.D. \& Rhazi L., 2009. Flore vasculaire endémique et menacée des zones humides de Tunisie. Revue d'Ecologie (Terre et Vie). 64, 19-40.

Guinochet M., 1973. Phytosociologie. Ed. Masson, 227p.

Koull N. \& Chehma A., 2013. Diversité floristique des zones humides de la vallée de l'oued Righ, (Sahra septentrional Algérien. -Revue des Bio-Ressources. Vol. 3 : 72-81.

Loisel R. \& Gomilla H., 1993. Traduction des effets du débroussaillement sur les écosystèmes forestiers et pré forestiers par un indice de perturbation. Annales de la Société des Sciences Naturelles et d'Archéologie de Toulon et du Var. 45 (2) : 123-132.

Journal officiel. 2012. Décret exécutif (n 12-03) Ministère d'Environnement. Algérie.

Maire R., 1957. Flore de l'Afrique du nord. -Volume IV et VIII. Ed. Chevalier. 336p.

Médail F. \& Quézel P., 1999. Biodiversity hotspots in the Mediterranean basin. - setting global conservation priorities. Conservation Biology.13: 1510-1513

Miara MD, Ait Hammou M. \& Hadjadj Aoul S., 2013. Bioclimats, Étages de Végétation et Zonation Altitudinale des Groupements Végétaux dans les Monts de Tiaret (Exemple du Massif de Guezoul). Revue Ecologie-Environnement : 9 : 7p.

Miara MD, Ait Hammou M, Hadjadj Aoul S, Dahmani W, Negadi M, Rebbas K, Bounar R. \& Smaili T., 2016. Notes sur les thérophytes dans les monts de Tiaret (massif de Guezoul-Algérie occidentale). Revue Écologie-Environnement. 12: 17-24.

Quézel P. \& Santa S., 1962. Nouvelle flore de l'Algérie et des régions désertiques méridionales. - C.N.R.S., 2 volumes, Paris. 1170 p.

Quézel P. \& Simonneau P., 1960. Quelques aspects de la végétation des terrains salés des plaines sublittorales de l'Oranie orientale.- Bulletin $n^{\circ} 6$. Direction de l'hydraulique et de l'équipement rural $29 \mathrm{p}$.

Simonneau P., 1952. Végétation halophile de la plaine de Perrégaux. -Gouvernement général de l'Algérie. Direction du service de l'hydraulique. - Ed, Clairbois- Birmandreis, Alger, 278 p.

Seltzer P., 1946. Le climat de l'Algérie. Carbonel. Alger, 219 p.

Ozenda P., 1977. Flore du Sahara. - Ed C.N.R.S. Paris. 623 p.

Tremblin G., 2000.Comportement autoécologique de Halopeplis amplexicaulis : Plante pionnière des Sebkhas de l'Ouest Algérien. Sécheresse. Vol. 11, 2 : 9-16.

Thomas G., 1976. Mise en évidence de décrochements dextre Est-Ouest d'âge Quaternaire en Algérie nord Occidentale, Comptes Rendus de l'Académie des Sciences de Paris, t. 283, 893896.

World Flora Online, 2020. www.worldfloraonline.org, consulté le 10/01/2020. 
Tableau 2 : Les halophytes des bassins fermés d'Oran (2012-2015)

$$
\text { (+ : présente, }- \text { : absente) }
$$

\begin{tabular}{|c|c|c|c|c|}
\hline $\begin{array}{l}\text { Les espèces récoltées autour des bassins fermés } \\
\text { d'Oran }\end{array}$ & Familles & $\begin{array}{l}\text { Typ. } \\
\text { Biol. }\end{array}$ & Chorologie & $\begin{array}{c}\text { Dubuis \& } \\
\text { Simonneau } \\
(\mathbf{1 9 6 0 )}\end{array}$ \\
\hline Mesembryanthemum nodiflorum $\mathrm{L}$. & AIZOACEAE & $\mathrm{Th}$ & Méd.-S.Afr. & + \\
\hline Mesembryanthemum cristalinum $\mathrm{L}$. & AIZOACEAE & Th & Méd.Aust., & - \\
\hline Arthrocnemum macrostachyum (Monic.) k. Koch & AMARANTHACEAE & $\mathrm{Ch}$ & Ancien Monde & + \\
\hline Atriplex canescens (Pursh) Nutt. & AMARANTHACEAE & $\mathrm{Ch}$ & Introduit & - \\
\hline Atriplex halimus L. & AMARANTHACEAE & $\mathrm{NPh}$ & Médit. et S.-afr. & + \\
\hline Atriplex prostrata DC. & AMARANTHACEAE & Th & Circumb. & + \\
\hline Atriplex patula $\mathrm{L}$ & AMARANTHACEAE & $\mathrm{Ch}$ & Circumb. & - \\
\hline Beta macrocarpa Guss. & AMARANTHACEAE & $\mathrm{Th}$ & Méd. & + \\
\hline Beta vulgaris subsp. maritima (L.) Arcang. & AMARANTHACEAE & Hé & Euras-Méd. & + \\
\hline Chenopodium album $\mathrm{L}$. & AMARANTHACEAE & Th & Cosmo., & - \\
\hline Chenopodium glaucum L. & AMARANTHACEAE & Th. & Adventice & - \\
\hline Halopeplis amplexicaulis (Vahl)Cesati \&al. & AMARANTHACEAE & Th & Méd. & + \\
\hline Sarcocornia perennis (Mill.) A.J. Scott & AMARANTHACEAE & $\mathrm{Ch}$ & Cosmo., & + \\
\hline Salicornia patula Duval-Jouve & AMARANTHACEAE & $\mathrm{Th}$ & Cosm. & + \\
\hline Salsola kali L. & AMARANTHACEAE & Th & Paléo-temp., & - \\
\hline Caroxylon vermiculatum (L.) Akhani \& Roalson & AMARANTHACEAE & $\mathrm{NPh}$ & Sah.Méd., & + \\
\hline Suaeda vera Forssk. ex J.F. Gmel. & AMARANTHACEAE & $\mathrm{Ch}$ & Cosmo., & + \\
\hline Suaeda maritima (L.) Dumort. & AMARANTHACEAE & Th & Cosmo., & + \\
\hline Bifora testiculata (L.) Spreng. & APIACEAE & $\mathrm{Th}$ & Méd. & - \\
\hline Bupleurum semicompositum L. & APIACEAE & Th & Méd. & + \\
\hline Daucus carota subsp. carota (L.) Thell. & APIACEAE & Hé & Méd., & + \\
\hline Foeniculum vulgare Mill. subsp. vulgare & APIACEAE & Gé & Méd & - \\
\hline Torilis nodosa (L.) Gaertn. & APIACEAE & Th & Euras. & + \\
\hline Arisarum vulgare Targ. Tozz. & ARACEAE & Gé & Circum-Méd. & - \\
\hline Asparagus horridus L. & ASPARAGACEAE & Gé & Macar.-Méd. & + \\
\hline Asphodelus ramosus L. & ASPHODELACEAE & Gé & Canar.Méd. & + \\
\hline Ajuga sp. & ASTERACEAE & $\mathrm{Ch}$ & - & + \\
\hline Anacyclus clavatus (Desf.) Pers & ASTERACEAE & Th & Eur.Méd. & + \\
\hline Anthemis pedunculata Desf. subsp. pedunculata & ASTERACEAE & Hé & Ibéro-Maur & - \\
\hline Atractylis cancellata $\mathrm{L}$. & ASTERACEAE & Th & Circum-Méd. & - \\
\hline Carlina gummifera (L.) Less. & ASTERACEAE & Hé & - & - \\
\hline Bellis annua L. subsp. annua & ASTERACEAE & $\mathrm{Th}$ & Circum-Méd. & + \\
\hline Calendula arvensis (Vaill.) & ASTERACEAE & $\mathrm{Th}$ & Sub-Méd. & + \\
\hline Calendula stellata Cav. & ASTERACEAE & Th & $\begin{array}{l}\text { can., sicile, } \\
\text { grece,Afr.sept. }\end{array}$ & - \\
\hline Cirsium palustre (L.) Scop. & ASTERACEAE & Hé & $\begin{array}{l}\text { Eur. moyenne et } \\
\text { sept. }\end{array}$ & - \\
\hline Catananche caerulea $\mathrm{L}$. & ASTERACEAE & Th & W. Méd & - \\
\hline Centaurea calcitrapa $\mathrm{L}$. & ASTERACEAE & Th. & Euryméd & - \\
\hline Rhaponticum coniferum (L.) Greuter & ASTERACEAE & Hé & W. Méd & - \\
\hline Centaurea pullata $\mathrm{L}$. & ASTERACEAE & Th & Méd. & - \\
\hline
\end{tabular}




\begin{tabular}{|c|c|c|c|c|}
\hline Centaurea sphaerocephala $\mathrm{L}$. & ASTERACEAE & Hé & Méd. & + \\
\hline Glebionis coronaria (L.) Spach & ASTERACEAE & $\mathrm{Th}$ & Méd. & + \\
\hline $\begin{array}{l}\text { Mauranthemum paludosum subsp. paludosum Poir. } \\
\text { Vog. \& Ober. }\end{array}$ & ASTERACEAE & Th. & Ibér. Maur. & - \\
\hline Cichorium intybus L.subsp. intybus & ASTERACEAE & Hé & $\operatorname{cosm}$ & + \\
\hline $\begin{array}{lll}\text { Picnomon acarna } & \text { (L.) Cass. }\end{array}$ & ASTERACEAE & Th & Méd. & - \\
\hline Erigeron canadensis L. & ASTERACEAE & $\mathrm{Th}$ & Amér. & - \\
\hline Filago pyramidata L. & ASTERACEAE & $\mathrm{Th}$ & Méd. & + \\
\hline pilosella pseudopilosella (Ten.) Sojak & ASTERACEAE & Th & Eur.Méd. & - \\
\hline Hypochaeris achyrophorus L. & ASTERACEAE & $\mathrm{Th}$ & Circum-Méd. & + \\
\hline Limbarda crithmoides (L.) Dumort. & ASTERACEAE & $\mathrm{Ch}$ & Méd. Atl., & + \\
\hline Dittrichia viscosa (L.) Greuter & ASTERACEAE & $\mathrm{Ch}$ & Circum-Méd. & + \\
\hline Matricaria chamomilla $\mathrm{L}$. & ASTERACEAE & $\mathrm{Th}$ & Euras.Macar. Mar. & - \\
\hline Otoglyphis pubescens (Desf.) Pomel & ASTERACEAE & Th. & Sah. & - \\
\hline Scolymus maculatus L. & ASTERACEAE & $\mathrm{Th}$ & Circum-Méd. & + \\
\hline $\begin{array}{l}\text { Senecio leucanthemifolius subsp. Mauritanicus } \\
\text { (Pomel) Greuter }\end{array}$ & ASTERACEAE & $\mathrm{Th}$ & Macar. Méd. & + \\
\hline Silybum marianum (L.) Gaertn. & ASTERACEAE & Th & $\operatorname{cosm}$ & - \\
\hline Sonchus mauritanicus Boiss. \& Reut & ASTERACEAE & Hé & End. Magreb & - \\
\hline Taraxacum erythrospermum Andrz. ex Besser & ASTERACEAE & $\mathrm{Th}$ & Méd & - \\
\hline Xanthium spinosum $\mathrm{L}$. & ASTERACEAE & Th & Subcosm & - \\
\hline Sonchus tenerrimus L. & ASTERACEAE & $\mathrm{Ch}$ & Méd. & + \\
\hline Anchusa hybrida Ten. & BORAGINACEAE & $\mathrm{Th}$ & Méd. & - \\
\hline Cynoglossum creticum Miller & BORAGINACEAE & Hé & Méd. & + \\
\hline Echium sabulicolum Pomel & BORAGINACEAE & $\mathrm{Th}$ & Méd. & + \\
\hline Capsella bursa-pastoris (L.) subsp. bursa-pastoris & BRASSICACEAE & Th & Méd. & - \\
\hline Eruca pinnatifida (Desf.) Pomel & BRASSICACEAE & Th & Méd. & - \\
\hline Eruca visicaria subsp. vesicaria (L.) Briq. & BRASSICACEAE & $\mathrm{Th}$ & Méd. & + \\
\hline Rorripa Nasturtium-aquaticum (L.) Hayek & BRASSICACEAE & Hé & $\operatorname{cosm}$ & - \\
\hline Sinapis arvensis L. & BRASSICACEAE & Th & Paléo-Temp. & + \\
\hline Arenaria emarginata Brot. & CARYOPHYLLACEAE & Th & Ibéro.Maur. & - \\
\hline Loefflingia hispanica (L.) subsp. hispanica & CARYOPHYLLACEAE & $\mathrm{Th}$ & Méd. & - \\
\hline Paronychia argentea Lam. & CARYOPHYLLACEAE & Hé & Méd. & + \\
\hline Silene gallica $\mathrm{L}$. & CARYOPHYLLACEAE & Th & Paléo-temp & - \\
\hline Silene nicaeensis All. & CARYOPHYLLACEAE & Th & Méd. & - \\
\hline Silene rubella $\mathrm{L}$. & CARYOPHYLLACEAE & $\mathrm{Th}$ & Méd & + \\
\hline Spergularia diandra (Guss.) Boiss. & CARYOPHYLLACEAE & Th & $\begin{array}{l}\text { Sah-Sind-Irano- } \\
\text { Tour. }\end{array}$ & - \\
\hline Spergularia doumerguaei P. Monnier & CARYOPHYLLACEAE & Th & End.Alg-Mar. & - \\
\hline Spergularia marina (L.) Besser & CARYOPHYLLACEAE & Th & Méd.-Stepp. & + \\
\hline $\begin{array}{l}\text { Convolvulus althaeoides subsp. tenuissimus (Sm.) } \\
\text { Batt. }\end{array}$ & CONVOLVULACEAE & Hé & Macar-Méd. & - \\
\hline Convolvulus lineatus L. & CONVOLVULACEAE & Hé & Méd. Asiat. & + \\
\hline Sedum rubens L. & CRASSULACEAE & Th & Méd. & - \\
\hline Ecballium elaterium Rich. & CUCURBITACEAE & Hé & Méd. & - \\
\hline Cynomorium coccineum L. subsp. Coccineum & CYNOMORIACEAE & Gé & Méd. & + \\
\hline Carex extensa Gooden & CYPERACEAE & Gé & Méd.-Austral.afr. & - \\
\hline Bolboschoenus maritimus (L.) Palla & CYPERACEAE & Gé & Cosm. & - \\
\hline
\end{tabular}




\begin{tabular}{|c|c|c|c|c|}
\hline Euphorbia segetalis subsp. pinea (L.) Rouy & EUPHORBIACEAE & $\mathrm{Th}$ & Méd.Atl. & - \\
\hline Lotus corniculatus L. subsp. corniculatus & FABACEAE & Hé & Eur.-As. & - \\
\hline Lotus creticus L. subsp. creticus Briquet & FABACEAE & $\mathrm{Ch}$ & Méd. & + \\
\hline Medicago italica (Mill.) Fiori & FABACEAE & $\mathrm{Th}$ & Méd & \\
\hline Medicago polymorpha $\mathrm{L}$. & FABACEAE & $\mathrm{Th}$ & Méd. & + \\
\hline Medicago laciniata (L.) Mill. & FABACEAE & Th & Méd.Sah.Sind & - \\
\hline Medicago dodiata Carmign & FABACEAE & $\mathrm{Th}$ & Méd. & - \\
\hline Melilotus indicus (L.) All. & FABACEAE & $\mathrm{Th}$ & Méd & + \\
\hline Melilotus infesta Guss & FABACEAE & Th & S.Méd. & - \\
\hline Melilotus sulcata Desf. & FABACEAE & $\mathrm{Th}$ & Méd. & + \\
\hline Scorpiurus muricatus (L.) subsp. muricatus Thell. & FABACEAE & Th & Méd. & - \\
\hline Trifolium tomentosum $\mathrm{L}$. & FABACEAE & Th. & Méd. & - \\
\hline Vicia sativa subsp. cordata (Hoppe) Battandier & FABACEAE & $\mathrm{Th}$ & Eur.-Méd. & - \\
\hline Frankenia corymbosa Desf. & FRANKENIACEAE & Hé & Méd. & + \\
\hline Frankenia hirsuta $\mathrm{L}$. & FRANKENIACEAE & $\mathrm{Ch}$ & Paléo-Temp. & - \\
\hline Frankenia pulverulenta L. subsp. pulverulenta & FRANKENIACEAE & Hé & Méd. & + \\
\hline Fumaria capreolata $\mathrm{L}$. & PAPAVERACEAE & Th & Méd. & - \\
\hline Centaurium tenuiflorum (Hoffm \& Link) Fritsch. & GENTIANACEAE & $\mathrm{Th}$ & Paléo-Temp. & + \\
\hline Centaurium erythraea Rafn. & GENTIANACEAE & Th & Eur.Méd. & - \\
\hline Erodium botrys (Cav.) Bertol. & GERANIACEAE & $\mathrm{Th}$ & Méd. & - \\
\hline Erodium ciconium (L.) L'Hér. & GERANIACEAE & $\mathrm{Th}$ & Méd. & - \\
\hline Erodium malacoides (L.) Willd. & GERANIACEAE & Th & Méd. & - \\
\hline Erodium laciniatum (Cav.) Willd. & GERANIACEAE & $\mathrm{Th}$ & Méd. & + \\
\hline Geranium lucidum (Bauhin) L. & GERANIACEAE & $\mathrm{Th}$ & Méd.-Atl. & - \\
\hline Geranium molle L. & GERANIACEAE & Th & Euras. & + \\
\hline Muscari comosum (L.) Mill. & HYACINTHACEAE & Gé & Méd. & - \\
\hline Juncus acutus L. subsp. acutus & JUNCACEEAE & Hé & Subcosm. & + \\
\hline Juncus maritimus Lamk. & JUNCACEEAE & Gé & Subcosm. & + \\
\hline Juncus subulatus forsk. & JUNCACEAE & Gé & Circum-Méd. & + \\
\hline Ballota hirsuta Benth. & LAMIACEAE & Th & Ibéro-Maur. & - \\
\hline Marrubium vulgare $\mathrm{L}$. & LAMIACEAE & $\mathrm{Ch}$ & Cosm. & + \\
\hline $\begin{array}{l}\text { Salvia phlomoides (Asso.) subsp. africana (M.), Greu.\& } \\
\text { Burdet }\end{array}$ & LAMIACEAE & Hé & Ibéro.-Maur & - \\
\hline Linum strictum $\mathrm{L}$. & LINACEAE & Th & Méd. & - \\
\hline Malva hispanica $\mathrm{L}$. & MALVACEAE & Hé & Ibéro.-Maur & - \\
\hline Malva neglecta Wallr. & MALVACEAE & Th & Méd. & - \\
\hline Malva sylvestris $\mathrm{L}$. & MALVACEAE & Hé & Euras & - \\
\hline Oxalis pres-caprae $\mathrm{L}$. & OXALIDACEEAE & Gé & le Cap & + \\
\hline Plantago macrorhiza Poir. & PLANTAGINACEAE & Hé & Euras. & + \\
\hline Plantago lagopus L. & PLANTAGINACEAE & Hé/Th & Méd. & + \\
\hline Plantago lanceolata (L.) subsp. lanceolata & PLANTAGINACEAE & Hé & Euras. & - \\
\hline Limonium cymuliferum (Boiss.) Sauv. \& Vindt & PLUMBAGINACEAE & Hé & End.Alg-Mar. & - \\
\hline Limonium duriaei (de Girard) Kuntze & PLUMBAGINACEAE & Hé & End.Alg-Mar. & + \\
\hline Limonium echioides (L.) Mill. & PLUMBAGINACEAE & Th & Méd. & + \\
\hline Limonium cossonianum Kuntze & PLUMBAGINACEAE & $\mathrm{Ch}$ & End. & - \\
\hline Limonium auriculaeursifolium (Pourr.) Druce & PLUMBAGINACEAE & Hé & $\begin{array}{l}\text { S. Eur., N Afr., Côte } \\
\text { Atl }\end{array}$ & - \\
\hline
\end{tabular}




\begin{tabular}{|c|c|c|c|c|}
\hline Limonium sinuatum subsp. sinuatum Sauvage \& Vindt. & PLUMBAGINACEAE & Hé & Méd.Sah.-Sind & + \\
\hline Limonium virgatum (Willd.) Fourr. & PLUMBAGINACEAE & Hé & Méd. & - \\
\hline Aegilops geniculata Roth subsp.geniculata & POACEAE & Th & Méd.-Irano-Tour & + \\
\hline Agrostis reuteri Boiss. & POACEAE & Hé & Ibéro-Maur. & \\
\hline Anisantha fasciculata (C. Presl) Nevski & POACEAE & Th & Paléo-subtrop & + \\
\hline Anisantha madritensis (L.) Nevski & POACEAE & Th & Eur. - Méd. & + \\
\hline Anisantha tectorum (L.) Nevski & POACEAE & $\mathrm{Th}$ & Paléotemp. & - \\
\hline Arundo donax L. & POACEAE & Gé & Méd. & + \\
\hline Avena fatua $\mathrm{L}$. & POACEAE & $\mathrm{Th}$ & Subcosm. & - \\
\hline Avena sterilis $\mathrm{L}$. & POACEAE & Th & $\begin{array}{l}\text { Macar.-Méd.-Irano- } \\
\text { Tour. }\end{array}$ & + \\
\hline Brachypodium retusum (Pers.) P. Beauv. & POACEAE & $\mathrm{Th}$ & Circum-Méd & - \\
\hline Bromus hordeaceus L. subsp. hordeaceus & POACEAE & Th & Paléotemp. & + \\
\hline Bromus lanceolatus subsp. lanceolatus Roth & POACEAE & $\mathrm{Th}$ & Paléotemp. & + \\
\hline Bromus squarrosus $\quad$ L. & POACEAE & $\mathrm{Th}$ & Paléotemp. & - \\
\hline Catapodium rigidum (L.) C. E. Hubb. & POACEAE & Th & Macar.-Euras.. & - \\
\hline $\begin{array}{l}\text { Corynephorus articulatus subsp. articulatus } \\
\text { (Asch.\&Gr.)Briq. }\end{array}$ & POACEAE & $\mathrm{Th}$ & Méd. & - \\
\hline Cynodon dactylon (L.) Pers. & POACEAE & Hé & Thermocosm. & + \\
\hline Dactilis glomerata $\mathrm{L}$. & POACEAE & Hé & Paléotemp. & + \\
\hline Elytrigia repens (L.) Desv. ex Nevski & POACEAE & Gé & Circumbor. & - \\
\hline Hordeum maritimum subsp. maritimum Huds. & POACEAE & $\mathrm{Th}$ & Méd.-Eur.-Amér. & + \\
\hline Hordeum murinum subsp. glaucum (Steud.) Tzvelev & POACEAE & Th & Circumbor. & - \\
\hline Lagurus ovatus L. & POACEAE & Th & Macar.- Méd. & + \\
\hline Lamarckia aurea (L.) Moench & POACEAE & Th & $\begin{array}{l}\text { Macar.-Méd.- } \\
\text { Ethiopie }\end{array}$ & - \\
\hline Lolium multiflorum Lam. & POACEAE & Hé & Méd. & + \\
\hline Lygeum spartum L. & POACEAE & Gé & W.Méd. & + \\
\hline Parapholis incurva (L.) C.E. Hubb. & POACEAE & $\mathrm{Th}$ & Méd. & + \\
\hline Phalaris canariensis L. & POACEAE & $\mathrm{Th}$ & Macar.- Méd. & - \\
\hline Phalaris paradoxa $\mathrm{L}$. & POACEAE & Th & Méd. & + \\
\hline $\begin{array}{c}\text { Phragmites australis (Cav.) Trin. ex Steud. } \\
\text { subsp. australis }\end{array}$ & POACEAE & -- & - & + \\
\hline Piptatherum miliaceum (L.) Coss. & POACEAE & Hé & Méd.-Irano-Tour. & + \\
\hline Polypogon monspeliensis (L.) Desf. & POACEAE & $\mathrm{Th}$ & paléo-subtrop. & + \\
\hline Rostraria cristata (L.) Tzvelev & POACEAE & $\mathrm{Th}$ & Sub-cosm & - \\
\hline Sphenopus divaricatus (Gouan) Rchb. & POACEAE & $\mathrm{Th}$ & paléo-subtrop. & + \\
\hline Polygonum maritimum L. & POLYGONACEAE & $\mathrm{Ch}$ & Cosm. & - \\
\hline Rumex pulcher L. & POLYGONACEAE & Hé & Méd. & - \\
\hline $\begin{array}{l}\text { Lysimachia arvensis f. parviflora (Hoff. \& Link) B. } \\
\text { Bock }\end{array}$ & PRIMULACEAE & Th & Sub.cosm. & + \\
\hline Reseda alba L. subsp. alba & RESEDACEAE & $\begin{array}{l}\text { Th/ } \\
\text { Hé }\end{array}$ & Euras & - \\
\hline Reseda alba L. subsp. hookeri & RESEDACEAE & $\begin{array}{l}\text { Th/ } \\
\text { Hé }\end{array}$ & Euras & - \\
\hline Galium aparine $\mathrm{L}$. & RUBIACEAE & Th & Paléo-temp. & - \\
\hline Hyoscyamus albus L. & SOLANACEAE & $\mathrm{Th}$ & Méd & - \\
\hline Lycium intricatum Boiss. & SOLANACEAE & $\mathrm{Ph}$ & Ibéro-Mar. & - \\
\hline Tamarix gallica L. & TAMARICACEAE & $\mathrm{ph}$ & N.Trop. & + \\
\hline
\end{tabular}




\begin{tabular}{|lcccc|}
\hline Tamarix africana Poiret & TAMARICACEAE & ph & W.Méd. & + \\
Thymelia hirsuta Endl. & TYMELIACEAE & $\mathrm{nph}$ & Méd. & - \\
Urtica dioica L. & URTICACEAE & Hé & Cosm & - \\
Urtica membranacea Poir. & URTICACEAE & Th & Méd & - \\
Urtica pilulifera L. & URTICACEAE & Th & Euras & - \\
Fedia cornucopiae (L.) Gaertn. & VALERIANACEAE & Th & Méd & - \\
\hline
\end{tabular}

\section{Abréviations utilisées dans le tableau 2:}

Types biologiques :

(Th) Thérophytes ; (He) Hémicryptophytes ; (Gé) Géophytes ; (Ch) Chaméphyte ; (NPh)

Nanophanérophytes ; $(\mathrm{Ph})$ Phanérophytes.

Chorologie :

Ensemble méditerranéen

Cent .-Méd. : Centre méditerranéenne

Circum-méd. : Circumméditerranéenne

E.- Med. : Est méditerranéenne

Ibéro.-Maur. : Ibéro-mauritanienne

Med. :Méditerranéenne

Oro.-Med:Oroméditerranéenne

W. Med. :Ouest méditerranéenne

End. :Endémique

End. A. N. : Endémique nord-africaine

End. Alg.-Mar. : Endémique algéro-marocaine

End. Alg.-Tun. : Endémique algéro-tunisienne

Mad. : Archipel de Madère (Portugal), inclus Ilhéus Salvages

Can. : Archipel des Îles Canaries (Espagne)

Atl.-Med. : Atlantique méditerranéenne

\section{Ensemble septentrionale}

Eur. : Européenne

Euras. : Eurasiatique

Paléo-temp. :Paléotempéré

Atl. : Atlantique

Circumbor. : Circumboréale

Euro.-Sib. : Eurosibérienne

Paléo.-bor. :Paléoboréale

W. Eur : Ouest européenne

Large répartition

Cosm. : Cosmopolite

Eur.-As. : Euro asiatique

Eur.-Med. : Euro méditerranéenne

Euras.-Med. : Eurasiatique méditerranéenne

Macar.-Euras. : Macaronésienne eurasiatique

Macar.-Med : Macaronésienne méditerranéenne

Med-As. : Méditerranéenne asiatique

Med- Irano-Tour. : Méditerranéo irano touranienne

Med-Sah.-Sind : Méditerranéo saharo sindienne

Pantropicale : taxon de toute la bande tropicale d'Eurasie, d'Afrique et d'Amérique. 